\title{
Incremento del módulo dinámico por efecto del densificado en ocho maderas mexicanas
}

\author{
Increase of the dynamic module due to the densified effect in eight Mexican woods
}

Javier Ramón Sotomayor-Castellanos ${ }^{1} \bullet$ Koji Adachi $^{\bullet}$ Ryuichi lida ${ }^{3} \bullet$ Tomoyuki Hayashi $^{4}$

\begin{abstract}
The objective of the research was to report the effects of densified by mechanical compression in woods of Cedrela odorata, Cupressus lindleyi, Swietenia macrophylla, Dalbergia paloescrito, Tabebuia donnell-smithii, Fraxinus uhdei, Fagus mexicana and Guazuma ulmifolia. The response variables were density and dynamic module, which was determined with transverse vibrations. Both parameters were measured before and after the treatment. The densified coefficient was, on average, $30 \%$ and similar between species. Both densities and dynamic modules increased as a result of densification. This technological gain is particular to each species. Although the results are restricted to densified species in this research, they suggest an improvement in these physical-mechanical characteristics that are important for the design and calculation of wood products and structures. To improve the treatment, studies on changes in wood anatomy and physical properties are recommended.
\end{abstract}

Key words: Wood density, densification coefficient, technological gain, Mexico

1. Universidad Michoacana de San Nicolás de Hidalgo, Morelia,

México; madera999@yahoo.com

2. Universidad Prefectoral de Akita, Noshiro, Japón;

kadachi@iwt.akita-pu.ac.jp

3. Universidad Politécnica en Tokio, Tokio, Japón;

Recibido: 11/09/2019

m111901m@st.u-gakugei.ac.jp

Aceptado: 19/11/2019

4. Universidad Prefectoral de Akita, Noshiro, Japón;

Publicado: 19/12/2019

tomoyuki_hayashi@iwt.akita-pu.ac.jp 


\section{Resumen}

El objetivo de la investigación fue informar sobre los efectos del densificado por compresión mecánica en maderas de Cedrela odorata, Cupressus lindleyi, Swietenia macrophylla, Dalbergia paloescrito, Tabebuia donnell-smithii, Fraxinus uhdei, Fagus mexicana y Guazuma ulmifolia. Las variables de respuesta fueron la densidad y el módulo dinámico, que fue determinado con vibraciones transversales. Ambos parámetros se midieron antes y después del tratamiento. El coeficiente de densificado fue, en promedio, de $30 \%$ y similar entre especies. Tanto las densidades como los módulos dinámicos aumentaron como efecto del densificado. Esta ganancia tecnológica es particular a cada especie. Si bien los resultados están restringidos a las especies densificadas en esta investigación, sugieren una mejora en estas características físico-mecánicas que son importantes para el diseño y cálculo de productos y estructuras de madera. Para mejorar el tratamiento se recomiendan estudios sobre los cambios en la anatomía de la madera y en sus propiedades físicas.

Key words: Densidad de la madera, coeficiente de densificado, ganancia tecnológica, México.

\section{Introducción}

La densidad de la madera es uno de sus principales rasgos funcionales [1] y se considera un buen predictor del módulo de elasticidad [2] . Igualmente, la densidad es la característica de referencia para pronosticar el módulo dinámico [3] e interviene en la configuración del índice de calidad para sugerir su uso como material de ingeniería [4]. La evidencia empírica sugiere que, a mayor densidad de la madera, se incrementa su módulo dinámico. Así, el paradigma contemporáneo en ciencias, tecnología e ingeniería de la madera propone que, si se aumenta de manera artificial su densidad, se obtendrá una ganancia tecnológica al aumentar su resistencia mecánica [5].

El densificado de la madera es el tratamiento por el cual su relación peso/volumen se incrementa al reducir los espacios vacíos en los lúmenes del tejido leñoso. Así, la madera densificada se define como aquella cuya densidad ha sido incrementada de manera artificial [6]. El densificado es un importante mecanismo de deformación que se presenta en varios procesos de manufactura de productos de madera reconstituida y de componentes estructurales [7]. Los tratamientos de densificado de la madera, de tipo termo mecánico, son de carácter eco-sustentable [8]. Esta tecnología se orienta hacia la diversificación de usos potenciales de especies de baja densidad natural y las provenientes de plantaciones de rápido crecimiento [9].

El método de vibraciones transversales es empleado para determinar el módulo dinámico de la madera densificada [10], [11], [12]. Los resultados de los autores citados indican que el módulo dinámico de la madera densificada aumenta comparativamente al de la madera sin densificar. Los factores que intervienen en este fenómeno son la especie, la temperatura, la intensidad de las deformaciones y el tiempo del densificado [5]. De aquí se sigue que es necesario comparar resultados de diferentes maderas densificadas con el mismo procedimiento. Aún más, para mejorar el uso de la madera como un material normalizado en ingeniería, es recomendable homogenizar los resultados experimentales.

Existe evidencia empírica de que el módulo dinámico de la madera aumenta a medida que su densidad se incrementa [2], [3], [4]. Este fenómeno se observa principalmente entre especies. Sin embargo, no existe información del incremento del módulo dinámico, determinado con vibraciones trasversales, cuando una misma madera se densifica. A manera de hipótesis de trabajo, la investigación propone que si se densifica por compresión mecánica la madera de Cedrela odorata L., Cupressus lindleyi Klotzsch ex Endl., Swietenia macrophylla King, Albergue paloescrito Rzed. \& GuridiGómez, Tabebuia donnell-smithii Rose, Fraxinus uhdei (Wenz.) Lingelsh., Fagus mexicana Martínez y Guazuma ulmifolia Lam, aumentan sus módulos dinámicos. Esta hipótesis puede verificarse si, antes y después de densificar su madera se evalúan las densidades y los módulos dinámicos son determinados con vibraciones transversales. El objetivo de la investigación es informar sobre el incremento en el módulo dinámico en ocho maderas mexicanas como efecto de su densificado.

\section{Materiales y métodos}

Las maderas fueron adquiridas en piezas aserradas en el estado de Michoacán, México. Para cada una de las especies, se recortaron veinte probetas con dimensiones de 0,02 $\mathrm{m} \times 0,02 \mathrm{~m} \times 0,4 \mathrm{~m}$ en las direcciones radial, tangencial y longitudinal respectivamente. Las probetas se almacenaron en una cámara de acondicionamiento con temperatura de $20{ }^{\circ} \mathrm{C}$ y humedad relativa del aire de $65 \%$ hasta que alcanzaron el contenido de humedad en equilibrio promedio de $10 \%$.

El contenido de humedad se calculó, después de las pruebas, con un grupo adicional de 20 muestras 
recortadas de un extremo de las probetas con dimensiones de 0,02 $\mathrm{m} \times 0,02 \mathrm{~m} \times 0,05 \mathrm{~m}$. El contenido de humedad se determinó por el método de diferencia de pesos de acuerdo a la norma ISO 13061-1:2014 [13], [14]. La densidad aparente de la madera se determinó con la fórmula (1):

$$
\rho_{\mathrm{CH}}=\frac{\mathrm{P}_{1}}{\mathrm{~V}_{1}}
$$

\section{Donde:}

$\mathrm{P}_{\mathrm{CH}}$ es la Densidad aparente de la madera para un contenido de humedad $\mathrm{CH}\left(\mathrm{kg} \mathrm{m}^{-3}\right)$

$\mathrm{P}_{1}$ es Peso de la probeta al momento del ensayo $(\mathrm{kg})$

$V_{1}$ es Volumen de la probeta al momento del ensayo $\left(\mathrm{m}^{3}\right)$

Para aligerar el texto, el término densidad sustituye al concepto densidad aparente. En el mismo orden de ideas, las siglas AD y DD se usan como indicadores de mediciones antes del densificado y después del densificado y el subíndice vt se refiere a mediciones y/o cálculos derivados de pruebas de vibraciones transversales.

El proceso de densificado consistió en cuatro etapas. Primeramente, las probetas se colocaron entre dos placas térmicas, con dimensiones de $1 \mathrm{~m} \times 1 \mathrm{~m}$, de una prensa Shon ai-Tekko, Modelo TS-100, con capacidad de 100 toneladas. La operación de prensado puede idealizarse como un sistema abierto y libre de restricción geométrica en la dirección transversal a la dirección de la carga. A continuación, las probetas de espesor inicial de 0,02 m se comprimieron en la dirección radial con una velocidad de prensado de $0,001 \mathrm{~m} \mathrm{~min}^{-1}$ hasta alcanzar un espesor objetivo de 0,014 m, equivalente a $70 \%$ de su dimensión original, que representa un coeficiente de densificado de 30 \% [15]. La presión aplicada varía de 30 a $50 \mathrm{MN} \mathrm{m}^{-2}$, dependiendo de la densidad de la madera. Posteriormente, las probetas en la prensa fueron calentadas a $120{ }^{\circ} \mathrm{C}$ durante 6 horas. Por último, las probetas fueron retiradas de la prensa y enfriadas a temperatura de laboratorio (20 $\left.{ }^{\circ} \mathrm{C}\right)$ durante 12 horas.

El ensayo de vibraciones transversales consistió en aplicar un impacto elástico en la dirección tangencial de la probeta, en el centro geométrico de su portada. La probeta estuvo apoyada en soportes simples, situados en los puntos nodales correspondientes al

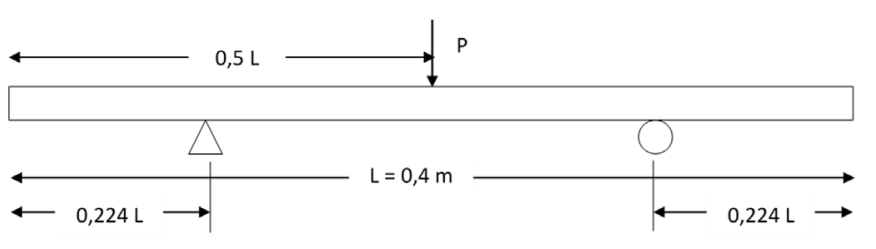

Figura 1. Ensayo de vibraciones transversales. $P=$ Impacto; $L=$ Longitud de la probeta.

Figure 1. Test of transverse vibrations. $P=\operatorname{Impact} ; \mathrm{L}=$ Length of the specimen.

primer modo de vibración (figura 1). De esta forma, la probeta fue solicitada en flexión transversal. Para el registro de la vibración de la probeta, se utilizó un sensor de movimiento de tipo piezoeléctrico. El sensor fue colocado a la mitad de la altura de la probeta y sobre un punto nodal, conectado al aparato Grindosonic MK5. Este sirvió para medir la frecuencia natural de la vibración.

El módulo de elasticidad dinámico se calculó con la fórmula (2) [16]:

$$
E_{v t}=\frac{4 \pi^{2} L_{v t}^{4} f_{v t}^{2} \rho_{C H}}{m^{4} r^{2}}\left(1+\frac{r^{2}}{I_{v t}^{2}} K\right)
$$

Donde:

$\mathrm{E}_{\mathrm{vt}}=$ Módulo de elasticidad dinámico $\left(\mathrm{N} \mathrm{m}^{-2}\right)$

$L_{v t}=$ Distancia de la portada entre apoyos $(\mathrm{m})$

$\mathrm{L}=$ Largo de la probeta $(\mathrm{m})$

$\mathrm{fvt}=$ Frecuencia natural de la probeta $(\mathrm{Hz})$

$\mathrm{P}_{\mathrm{CH}}=$ Densidad de la madera a un contenido de humedad $\mathrm{CH}\left(\mathrm{kg} \mathrm{m}^{-3}\right)$

$\mathrm{m}, \mathrm{K}=$ Constantes (adimensionales)

$r=$ Radio de giro de la sección transversal de la probeta $\left(\mathrm{m}^{2}\right)$

Para medir el efecto del tratamiento en las magnitudes de las densidades y módulos dinámicos, se calcularon sus incrementos con la fórmula (3):

$$
\Delta=\frac{M_{D D}-M_{A D}}{M_{A D}} \times 100
$$

Donde:

$\Delta=$ Incremento (\%) 
Cuadro 1. Densidades y módulos dinámicos antes y después del densificado.

Table 1. Densities and dynamic modulus before and after densification.

\begin{tabular}{|c|c|c|c|c|c|}
\hline \multirow{2}{*}{ Especies } & \multirow{2}{*}{ Código } & $\mathrm{P}_{\mathrm{CH}} \mathrm{AD}$ & $E_{v t} A D$ & $\mathrm{P}_{\mathrm{CH}} \mathrm{DD}$ & $E_{v t} D D$ \\
\hline & & $\left(\mathrm{kg} \mathrm{m}^{-3}\right)$ & $\left(\mathrm{MN} \mathrm{m}^{-2}\right)$ & $\left(\mathrm{kg} \mathrm{m}^{-3}\right)$ & $\left(\mathrm{MN} \mathrm{m}^{-2}\right)$ \\
\hline Cupressus lindleyi & $\mathrm{CL}$ & $461(12,1)$ & $12790(21,4)$ & $607(11,4)$ & $29381(18,3)$ \\
\hline Cedrela odorata & $\mathrm{CO}$ & $473(19,5)$ & $11327(14,4)$ & $743(6,2)$ & $26324(14,7)$ \\
\hline Swietenia macrophylla & SM & $540(6,7)$ & $10443(19,2)$ & $743(6,2)$ & $25039(19,4)$ \\
\hline Tabebuia donnell-smithii & $\mathrm{TD}$ & $602(7,1)$ & $8834(12,0)$ & $783(5,4)$ & $19248(7,7)$ \\
\hline Fraxinus uhdei & FU & $611(4,8)$ & $10121(9,5)$ & $823(3,8)$ & $24214(8,5)$ \\
\hline Fagus mexicana & FM & $621(5,2)$ & $10062(9,1)$ & $853(5,4)$ & $22639(8,2)$ \\
\hline Dalbergia paloescrito & DP & $662(10,2)$ & $12298(21,2)$ & $878(8,7)$ & $29788(21,5)$ \\
\hline Guazuma ulmifolia & GU & $675(6,0)$ & $7830(10,1)$ & $920(5,7)$ & $19091(8,3)$ \\
\hline
\end{tabular}

$\mathrm{P}_{\mathrm{CH}}=$ Densidad; $\mathrm{E}_{\mathrm{vt}}=$ Módulo dinámico; $\mathrm{AD}$ = Antes del densificado; $\mathrm{DD}$ = Después del densificado; $(\mathrm{CV})=$ Coeficiente de variación en porciento; Códigos para las figuras 2 y 3 .

MDD = Magnitud después del densificado

MAD = Magnitud antes del densificado

\section{Diseño experimental}

Cada grupo de probetas de las ocho especies se consideró una muestra independiente. En lo subsecuente y cuando conviene, para aligerar la lectura, el término tratamiento hace referencia al tratamiento de densificado. El tratamiento se consideró el factor de variación. Las variables de respuesta fueron la densidad y el módulo dinámico. Para cada una de las variables de respuesta se efectuaron pruebas con 20 réplicas (probetas por especie), por lo que en total se realizaron 160 observaciones. El contenido de humedad fue un parámetro de referencia.

Se realizaron pruebas de normalidad de la distribución de las muestras y se calcularon los sesgos y apuntamientos estandarizados, así como las medias, las desviaciones estándar y los coeficientes de variación. También, se efectuaron pruebas de verificación y análisis de varianza para un nivel de confianza de $95 \%$. Cuando las distribuciones no fueron normales se llevaron a cabo pruebas de Kruskal-Wallis. Asimismo, para medir la intensidad de la asociación entre las variables antes y después del densificado, se evaluaron correlaciones lineales entre ellas.

\section{Resultados y discusión}

Las magnitudes de las densidades y módulos dinámicos, así como sus respectivos coeficientes de variación (cuadro 1), fueron similares a los valores para maderas mexicanas de densidad comparable, presentados por [17], [18], [19]. Las pruebas de normalidad indicaron que las distribuciones de los módulos dinámicos de $C$. odorata (AD) y S. macrophylla (DD) no satisfacen los criterios de demarcación para aceptar sus distribuciones normales (cuadro 2). Las pruebas de verificación y análisis de varianza, tanto de las densidades como de los módulos dinámicos de las ocho maderas, sugieren diferencias estadísticamente significativas entre las mediciones antes y después del tratamiento (cuadro 2).

Las regresiones entre las densidades y módulos dinámicos después del densificado, en función de estos mismos parámetros medidos antes del tratamiento, resultaron con coeficientes de determinación significativos (figuras 2 y 3 ). Sus magnitudes $(0,86<$ $\left.\mathrm{R}^{2}<0,95\right)$ fueron representativas de los reportados para maderas mexicanas [17], [18], [19]. En efecto, los coeficientes de determinación del modelo lineal explicaron, con una calidad de ajuste satisfactorio, entre el 86 y $95 \%$ de la variación ocasionada por el tratamiento de densificado. Esta ganancia tecnológica indicada por los incrementos en las densidades y módulos dinámicos es particular a cada especie.

Las densidades de la madera densificada, respecto a las magnitudes antes del densificado, se incrementaron en promedio $37 \%$. Igualmente, los módulos dinámicos aumentaron $134 \%$. De manera más detallada, se observó que los incrementos en los valores de las densidades de 


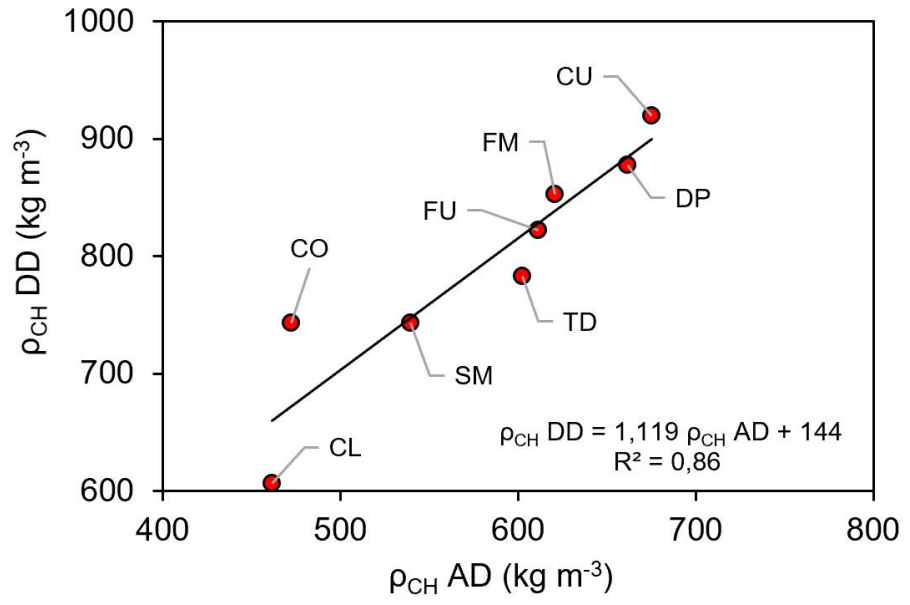

Fugura 2. Correlación entre densidades $\left(P_{C H}\right)(A D)$ y (DD). Códigos del Cuadro 1.

Figure 2. Correlation between densities $\left(P_{C H}\right)(A D)$ and $(D D)$. Codes from table 1.

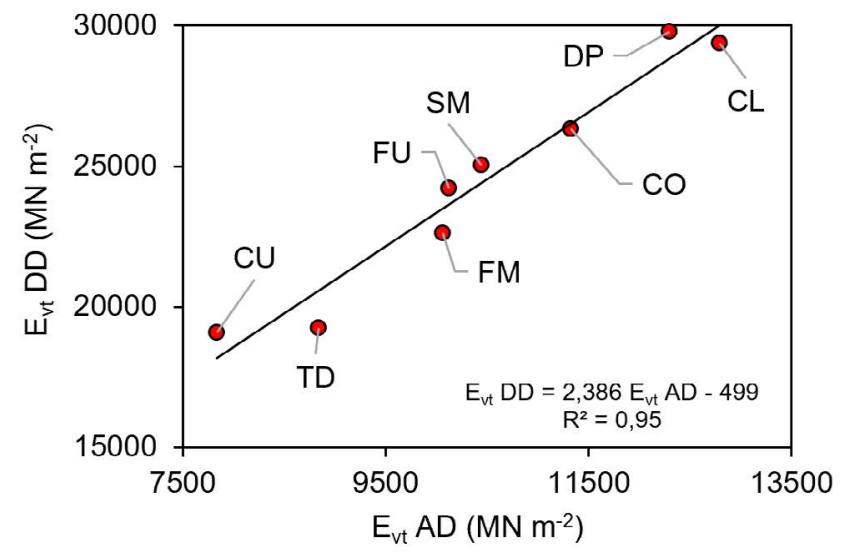

Figura 3. Correlación entre módulos dinámicos $\left(E_{v t}\right)(A D)$ y (DD). Códigos del Cuadro 1.

Figure 3. Correlation between dynamic modulus $\left(E_{v t}\right)(A D)$ and (DD). Codes from table

siete especies se agrupan en el intervalo de un mínimo de $30 \%$ para $T$. donnell-smithii y un máximo de $38 \%$ para S. macrophylla. Solamente $C$. odorata se distingue con un incremento de $57 \%$. Por su parte, los incrementos en los módulos dinámicos se agrupan de manera uniforme entre un mínimo de $118 \%$ para $T$. donnell-smithii y un máximo de $144 \%$ para G. ulmifolia. Estos resultados se detallan en la figura 4.

Kutnar, Kamke y Sernek [20], Skyba, Schwarze y Niemz [21] y Ulker y Hiziroglu [22] reportan que los resultados

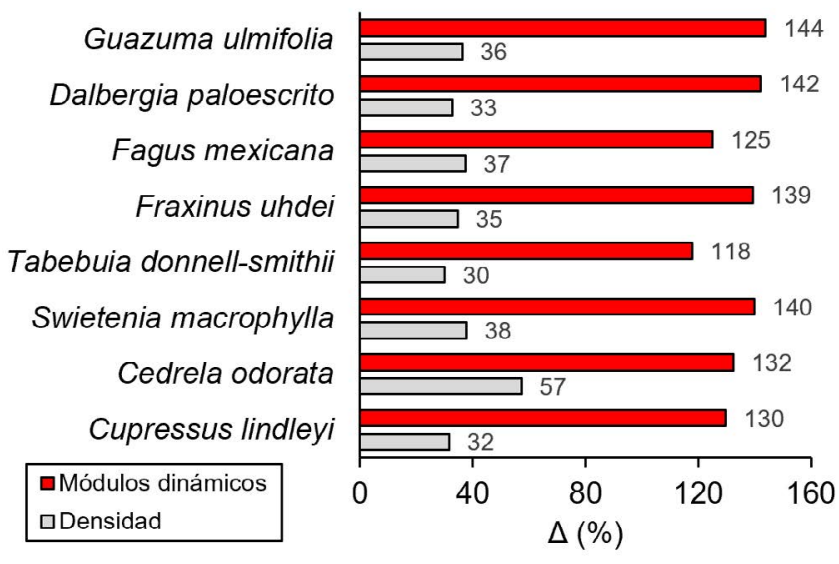

Figura 4. Incrementos $(\Delta)$ en la densidad $\left(P_{C H}\right)$ y en los módulos dinámicos $\left(\mathrm{E}_{\mathrm{vt}}\right)$.

Figure 4. Increases $(\Delta)$ in density $\left(P_{C H}\right)$ and in dynamic modulus $\left(E_{v t}\right)$.

empíricos son diferentes para cada caso de estudio y según las condiciones del tratamiento. Entre los parámetros que explican las propiedades finales de la madera densificada [23], [24], [25] están la densidad y morfología de cada especie, la temperatura, el tiempo y la presión en el tratamiento de densificado. Si bien en la presente investigación la estrategia experimental fue observar únicamente la variación entre especies, la diversidad entre resultados coincide con la reportada en la bibliografía. Por ejemplo, empleando procesos de densificación higro-termo-mecánicos, [23] densifican madera de Pinus sylvestris y reportan incrementos en la densidad de $93 \%$ y en su módulo de elasticidad de $114 \%$. Por su parte, [24] reportan que después del densificado de Populus tremuloides su densidad aumenta $97 \%$ y su módulo de elasticidad se incrementa $60 \%$. En el mismo contexto, para madera densificada de Populus tomentosa, [25] reportan un incremento en la densidad de $100 \%$ y un incremento en el módulo de elasticidad de $73 \%$.

El aumento artificial de la densidad de la madera incrementa la resistencia mecánica, mejora sus propiedades higroscópicas y aumenta su resistencia al ataque de agentes biológicos. No obstante, si la madera densificada se expone a la humedad, tiende a recuperar su forma original [26]. Este fenómeno de recuperación geométrica de la madera densificada no es equivalente al fenómeno de higroexpansión de la madera. En el caso de la madera densificada, es el resultado de la acción de la humedad, combinado con el efecto de la liberación de esfuerzos incorporados durante los procesos de deshumidificado, calentado y deformado propios del densificado [27]. 
Cuadra 2. Resultados pruebas de normalidad y de hipótesis.

Table 2. Normality and hypothesis test results

\begin{tabular}{|c|c|c|c|c|c|c|c|}
\hline & \multicolumn{4}{|c|}{ Pruebas de normalidad } & \multicolumn{3}{|c|}{ Pruebas de hipótesis } \\
\hline & $A D$ & & $\mathrm{DD}$ & & Ver-var & Anova & $\mathrm{K}-\mathrm{W}$ \\
\hline & SE & $\mathrm{AE}$ & SE & $\mathrm{AE}$ & $P(a=0,05)$ & $P(a=0,05)$ & $P(a=0,05)$ \\
\hline & \multicolumn{7}{|c|}{ Cupressus lindleyi } \\
\hline $\mathrm{P}_{\mathrm{CH}}$ & 1,274 & $-0,321$ & 1,648 & 0,663 & 0,503 & $<0,001^{*}$ & $<0,001^{*}$ \\
\hline \multirow[t]{2}{*}{$E_{v t}$} & 1,036 & $-1,033$ & 0,445 & $-0,793$ & 0,012 & $<0,001$ & $<0,001$ \\
\hline & \multicolumn{7}{|c|}{ Cedrela odorata } \\
\hline $\mathrm{P}_{\mathrm{CH}}$ & $-0,533$ & $-1,181$ & $-0,277$ & 1,247 & 0,000 & $<0,001$ & $<0,001$ \\
\hline \multirow{2}{*}{$\mathrm{E}_{\mathrm{vt}}$} & $-2,186$ & 0,703 & $-1,776$ & 0,092 & 0,017 & $<0,001$ & $<0,001$ \\
\hline & \multicolumn{7}{|c|}{ Swietenia macrophylla } \\
\hline $\mathrm{P}_{\mathrm{CH}}$ & 0,316 & 0,714 & $-0,277$ & 1,247 & 0,569 & $<0,001$ & $<0,001$ \\
\hline \multirow[t]{2}{*}{$\mathrm{E}_{\mathrm{vt}}$} & $-1,544$ & 0,298 & $-0,943$ & 2,473 & 0,035 & $<0,001$ & $<0,001$ \\
\hline & \multicolumn{7}{|c|}{ Tabebuia donnell-smithii } \\
\hline $\mathrm{P}_{\mathrm{CH}}$ & 0,584 & $-0,895$ & 0,702 & $-0,372$ & 0,566 & $<0,001$ & $<0,001$ \\
\hline \multirow[t]{2}{*}{$E_{v t}$} & 0,125 & $-0,648$ & $-0,617$ & $-1,024$ & 0,141 & $<0,001$ & $<0,001$ \\
\hline & \multicolumn{7}{|c|}{ Fraxinus uhdei } \\
\hline$P_{C H}$ & $-0,841$ & $-0,814$ & $-0,274$ & $-0,234$ & 0,896 & $<0,001$ & $<0,001$ \\
\hline \multirow[t]{2}{*}{$E_{v t}$} & $-1,232$ & $-0,199$ & $-1,184$ & 0,946 & 0,080 & $<0,001$ & $<0,001$ \\
\hline & \multicolumn{7}{|c|}{ Fagus mexicana } \\
\hline $\mathrm{P}_{\mathrm{CH}}$ & $-0,259$ & $-0,935$ & 0,542 & $-0,839$ & 0,120 & $<0,001$ & $<0,001$ \\
\hline \multirow[t]{2}{*}{$E_{v t}$} & $-0,550$ & $-0,375$ & $-1,077$ & $-0,177$ & 0,019 & $<0,001$ & $<0,001$ \\
\hline & \multicolumn{7}{|c|}{ Dalbergia paloescrito } \\
\hline $\mathrm{P}_{\mathrm{CH}}$ & 0,674 & $-0,103$ & 0,127 & 0,352 & 0,766 & $<0,001$ & $<0,001$ \\
\hline \multirow[t]{2}{*}{$E_{v t}$} & 1,444 & $-0,289$ & 1,024 & $-0,561$ & 0,002 & $<0,001$ & $<0,001$ \\
\hline & \multicolumn{7}{|c|}{ Guazuma ulmifolia } \\
\hline $\mathrm{P}_{\mathrm{CH}}$ & 0,876 & $-1,003$ & 0,748 & $-0,621$ & 0,404 & $<0,001$ & $<0,001$ \\
\hline$E_{v t}$ & 0,982 & 1,143 & 1,347 & 1,358 & 0,034 & $<0,001$ & $<0,001$ \\
\hline
\end{tabular}

Los resultados de la investigación son particulares a la madera de las especies densificadas con el tratamiento utilizado en esta investigación. Para mejorar el tratamiento de densificado se recomienda realizar estudios sobre los cambios en la anatomía de la madera e identificar en qué medida se modifican su estructura y sus propiedades físicas.

\section{Conclusiones}

Se densificó madera de C. odorata, C. lindleyi, S. macrophylla, D. paloescrito, T. donnell-smithii, F. uhdei, F. mexicana y G. ulmifolia y se determinaron antes y después del tratamiento de densificado, sus densidades y módulos dinámicos con vibraciones transversales.
Las densidades y los módulos dinámicos aumentan como efecto del densificado. Esta ganancia tecnológica es particular a cada especie. El coeficiente de densificado fue en promedio de $30 \%$ y similar entre especies, resultado que sugiere una mejora en estas características físico-mecánicas importantes para el diseño y cálculo de productos y estructuras de madera.

\section{Agradecimentos}

La investigación estuvo patrocinada por la Universidad Michoacana de San Nicolás de Hidalgo, México y por la 
Universidad Prefectoral de Akita, Japón.

\section{Referências}

[[1] J. Dlouhá, T. Alméras, J. Beauchêne, B. Clair, y M. Fournier, "Biophysical dependences among functional wood traits", Functional Ecology, vol. 32, no. 12, pp. 2652-2665, 2018.

[2] K. J. Niklas, y H. C. Spatz, "Worldwide correlations of mechanical properties and green wood density", American Journal of Botany, vol. 97, no. 10, pp. 1587-1594, 2010.

[3] I. Brémaud, Y. El Kaïm, D. Guibal, K. Minato, T. Thibaut, y J. Gril, "Characterisation and categorisation of the diversity in viscoelastic vibrational properties between 98 wood types", Annals Forest Science, vol. 69, no. 3, pp. 373-386, 2012.

[4] U. G. K. Wegst, "Wood for sound", American Journal of Botany, vol. 93, no. 10, pp. 1439-1448, 2006.

[5] A. Kutnar, y M. Šernek, "Densification of wood". Zbornik gozdarstva in lesarstva, vol. 82, pp. 53-62, 2007.

[6] D. Sandberg, y P. Navi, Introduction to Thermo-hydromechanical (THM) Wood Processing. Växjö, Sweden: Växjö University, 2007.

[7] Y. E. Aimene, y J. A. Nairn, "Simulation of transverse wood compression using a large-deformation, hyperelasticplastic material model", Wood Science and Technology, vol. 49, no. 1, pp. 21-39, 2015.

[8] D. Sandberg, P. Haller, y P. Navi, "Thermo-hydro and thermohydro-mechanical wood processing: An opportunity for future environmentally friendly wood products", Wood Material Science \& Engineering, vol. 8, no. 1, pp. 64-88, 2013.

[9] C. P. Gabrielli, y F. A. Kamke, "Phenol-formaldehyde impregnation of densified wood for improved dimensional stability", Wood Science and Technology, vol. 44, no. 1, pp. 95-104, 2010.

[10] S. Hayashi, y H. Nishimura, "Study on static \& dynamic bending strength of compressed lumber and the utilization for reinforcement", Transactions of the Japan Society of Mechanical Engineers Series A, vol. 67, no. 656, pp. 757762, 2001.

[11] Y. Kubojima, T. Ohtani, y H. Yoshihara, "Effect of shear deflection on vibrational properties of compressed wood", Wood Science and Technology, vol. 38, no. 3, pp. 237244, 2004.

[12] S. Chen, E. Obataya, y M. Matsuo-Ueda, "Shape fixation of compressed wood by steaming: a mechanism of shape fixation by rearrangement of crystalline cellulose", Wood Science and Technology, vol. 52, no. 5, pp. 1229-1241, 2018.

[13] ISO (International Standards Organization), Physical and mechanical properties of wood. Test methods for small clear wood specimens. Part 1: Determination of moisture content for physical and mechanical tests, ISO 130611:2014, 2014.

[14] ISO (International Standards Organization), Physical and mechanical properties of wood. Test methods for small clear wood specimens. Part 2: Determination of density for physical and mechanical tests, ISO 13061-2:2014, 2014.

[15] J. Blomberg, B. Persson, y A. Blomberg, "Effects of semiisostatic densification of wood on the variation in strength properties with density", Wood Science and Technology, vol. 39, no. 5, pp. 339-350, 2005.

[16] L. Machek, H. Militz y R. Sierra-Alvarez, "The use of an acoustic technique to assess wood decay in laboratory soil-bed tests", Wood Science and Technology, vol. 34, no. 6, pp. 467-472, 2001.

[17] J. A. Silva, F. J. Fuentes, R. Rodríguez, P. A. Torres, M. A. Lomelí, J. Ramos, C. Waitkus, y H. G. Richter, Fichas de propiedades tecnológicas y usos de maderas nativas de México e importadas. México: Comisión Nacional Forestal, 2010.

[18] J. R. Sotomayor, Banco FITECMA de características físicomecánicas de maderas mexicanas. Morelia: Universidad Michoacana de San Nicolás de Hidalgo, 2015.

[19] C. Tamarit y J. L. López, Xilotecnología de los principales árboles tropicales de México. México: Instituto Nacional de Investigaciones Forestales, Agrícolas y Pecuarias, 2007.

[20] A. Kutnar, F. A. Kamke, y M. Sernek, "The mechanical properties of densified VTC wood relevant for structural composites", Holz als Roh- und Werkstoff, vol. 66, no. 6, pp. 439-446, 2008.

[21] O. Skyba, F. Schwarze, y P. Niemz, "Physical and Mechanical Properties of Thermo-Hygro-Mechanically (THM)Densified Wood", Wood Research, vol. 54, no. 2, pp. 1-18, 2009.

[22] O. Ulker, y S. Hiziroglu, "Some Properties of Densified Eastern Redcedar as Function of Heat and Pressure", Materials, vol. 10, no. 11, pp. 1275-1289, 2017.

[23] O. Ulker, O. Imirzi, y E. Burdurlu, "The effect of densification temperature on some physical and mechanical properties of scots pine (Pinus sylvestris L.)", Bioresources, vol. 7, no. 4, pp. 5581-5592, 2012.

[24] C. H. Fang, A. Cloutier, P. Blanchet, y A. Koubaa, "Densification of wood veneers combined with oil heat treatment. Part II: hygroscopicity and mechanical properties", Bioresources, vol. 7, no. 1, pp. 925-935, 2012.

[25] Z. Gao, R. Huang, J. Lu, Z. Chen, F. Guo, y T. Zhan, "Sandwich compression of wood: control of creating density gradient on lumber thickness and properties of compressed wood", Wood Science and Technology, vol. 50, no. 4, pp. 833844, 2016.

[26] M. J. Boonstra, y J. Blomberg, "Semi-isostatic densification of heat-treated radiata pine", Wood Science and Technology, vol. 41, no. 7, pp. 607-617, 2007.

[27] P. Navi, y F. Heger, F, "Combined Densification and ThermoHydro-Mechanical Processing of Wood”, MRS Bulletin, vol. 29, no. 5, pp. 332-336, 2004. 